\title{
Found in Translation: High-Throughput Chemical Screening in Arabidopsis thaliana Identifies Small Molecules That Reduce Fusarium Head Blight Disease in Wheat
}

\author{
Karl J. Schreiber, ${ }^{1}$ Charles G. Nasmith, ${ }^{2}$ Ghislaine Allard, ${ }^{2}$ Jasbir Singh, ${ }^{2}$ Rajagopal Subramaniam, ${ }^{2}$ \\ and Darrell Desveaux ${ }^{1,3}$ \\ ${ }^{1}$ Department of Cell \& Systems Biology, University of Toronto, 25 Willcocks St., Toronto, Ontario M5S 3B2, Canada; \\ ${ }^{2}$ Agriculture and Agri-Food Canada/Agriculture et Agroalimentaire Canada, KW Neatby Bldg., 960 Carling Ave., Ottawa, \\ Ontario K1A 0C6, Canada; ${ }^{3}$ Centre for the Analysis of Genome Evolution \& Function, University of Toronto
}

Submitted 16 September 2010. Accepted 28 January 2011.

\begin{abstract}
Despite the tremendous economic impact of cereal crop pathogens such as the fungus Fusarium graminearum, the development of strategies for enhanced crop protection is hampered by complex host genetics and difficulties in performing high-throughput analyses. To bypass these challenges, we have developed an assay in which the interaction between $F$. graminearum and the model plant Arabidopsis thaliana is monitored in liquid media in 96-well plates. In this assay, fungal infection is associated with the development of dark lesion-like spots on the cotyledons of Arabidopsis seedlings by 4 days postinoculation. These symptoms can be alleviated by the application of known defense-activating small molecules and in previously described resistant host genetic backgrounds. Based on this infection phenotype, we conducted a small-scale chemical screen to identify small molecules that protect Arabidopsis seedlings from infection by $\boldsymbol{F}$. graminearum. We identified sulfamethoxazole and the indole alkaloid gramine as compounds with strong protective activity in the liquid assay. Remarkably, these two chemicals also significantly reduced the severity of $F$. graminearum infection in wheat. As such, the Arabidopsisbased liquid assay represents a biologically relevant surrogate system for high-throughput studies of agriculturally important plant-pathogen interactions.
\end{abstract}

The ascomycete fungus Fusarium graminearum (teleomorph Gibberella zeae (Schwein.) Petch) is an economically significant pathogen of cereal crops such as wheat, barley, maize, oat, and rye (Parry et al. 1995). The development of Fusarium head blight disease on these crops generally occurs during warm days and cool nights, particularly in spring, when susceptible host plants are flowering. Infection occurs at anthesis, when rain-splashed conidia or airborne ascospores from infected crop debris contact and germinate on floral tissues. Upon invasion of these tissues, the fungus proliferates and spreads to neighboring spikelets within a spike (Goswami and Kistler 2004). Subsequent necrosis of infected tissues is associated with reductions in both grain yield and quality. Infection is also accompanied by the in planta accumulation of various tri-

Correspondence authors:

R. Subramaniam; E-mail; rajagopal.subramaniam@agr.gc.ca and

D. Desveaux: darrell.desveaux@utoronto.ca

* The $\boldsymbol{e}$-Xtra logo stands for "electronic extra" and indicates that four supplementary tables and one supplementary figure are published online. cothecene mycotoxins by $F$. graminearum, including nivalenol, deoxynivalenol (DON), 3-acetyl deoxynivalenol (3-ADON), and 15-acetyl deoxynivalenol (15-ADON) (Hohn et al. 1998). These toxins inhibit protein translation (Cundliffe et al. 1974) and represent a serious health issue when present at high concentrations. In terms of resistance to $F$. graminearum infection, the primary mechanisms involve resistance to initial infection (type I), resistance to fungal spread within an infected spike (type II), and reduced DON accumulation (type III) (Bai and Shaner 2004). Presently, a limited number of wheat genotypes are known to confer type II resistance (Anderson et al. 2001; Ban and Suenaga 2000; Cuthbert et al. 2006; Van Ginkel et al. 1996); however, the molecular events underlying this resistance remain unknown.

The genetic complexity of polyploid cereals presents a considerable challenge for the characterization of resistance to $F$. graminearum, such that the use of a more genetically tractable host such as Arabidopsis represents an appealing alternative. Previous investigations of the Arabidopsis- $F$. graminearum interaction involved a range of inoculation techniques, including pressure infiltration of leaf tissue (Makandar et al. 2006), point inoculation of wounded siliques (Cuzick et al. 2008), spray inoculation of floral organs (Urban et al. 2002), and wound inoculation of detached leaves (Chen et al. 2006). Although reproducible and biologically relevant data can be obtained with these protocols, they are difficult to extend to high-throughput assays. We previously developed an assay in which Arabidopsis seedlings are grown in liquid media in 96-well plates (Schreiber et al. 2008). We demonstrated that this experimental setup could be used to study the Arabidopsis-Pseudomonas syringae pathosystem with great economy of both time and space. In particular, this assay was amenable to high-throughput chemical screens to identify small molecules that alter the outcome of the Arabidopsis $-P$. syringae interaction. The chemical genetic approach provides several advantages over classical forward genetic screening (Kawasumi and Nghiem 2007; Stockwell 2000), including the capability to perturb biological systems at the proteome level. Importantly, given that the structure of protein families is often more highly conserved than are their primary sequences (Illergård et al. 2009), the activity of a compound in a model system should translate to other systems. Furthermore, many known defense-inducing molecules are active in a broad range of host plants (Schreiber and Desveaux 2008).

In this study, we characterize the Arabidopsis-F. graminearum interaction in a liquid-based assay. We demonstrate that this interaction can be modulated by the application of chemicals, 
either to "prime" host defenses or to directly inhibit pathogen growth. Based on these phenotypic observations, we initiated a screen to identify small molecules that protect Arabidopsis seedlings from infection by $F$. graminearum. Importantly, the protective activity of compounds of interest was subsequently established in wheat. Such translation to a native host of $F$. graminearum validates the biological relevance of the Arabidopsis screening assay.

\section{RESULTS}

\section{Characterization of the Arabidopsis-F. graminearum pathosystem in liquid media.}

In order to evaluate the Arabidopsis- $F$. graminearum interaction in a high-throughput manner, we examined this pathosystem using an assay conducted in liquid media in 96-well plates (Schreiber et al. 2008). In this environment, inoculation of Arabidopsis seedlings with $F$. graminearum spores led to a highly reproducible progression of macroscopic disease symptoms (Fig. 1). In particular, small brown spots were visible on cotyledons within 2 days postinoculation (dpi). Over time, these spots increased in both size and number and, by $4 \mathrm{dpi}$, any cotyledonary tissues that lacked spots were nearly translu- cent. Hypocotyls remained relatively unaffected by the fungus, and root tips exhibited a general browning over time. Trypan blue staining revealed numerous sites of cell death in cotyledons at 2 dpi, especially at the tips of these tissues (Fig. 1F to J). Notably, localized sites of staining were generally centered at or near stomatal guard cells (Fig. 1H). As with the macroscopic spotting symptoms, sites of trypan blue staining became more abundant and widespread as time progressed. By 4 dpi, however, many cotyledons exhibited reduced staining of plant cells, while fungal hyphae were especially prominent, having now completely innervated most cotyledonary tissues (Fig. $1 \mathrm{~J})$. Inoculation of wells with green fluorescent protein (GFP)expressing $F$. graminearum clearly illustrated the extensive fungal invasion of cotyledons at 4 dpi (Fig. $1 \mathrm{~K}$ and L). By this point, a mass of fungal mycelia was also evident in inoculated wells (data not shown). Interestingly, a DON-deficient $\Delta$ tri6 mutant of $F$. graminearum elicited disease symptoms very similar to those of the wild-type fungus, indicating that DON is dispensable for infection of Arabidopsis seedlings in liquid media (Fig. 1M and N).

The infection symptoms depicted in Figure 1 could be significantly attenuated by altering host defense responses either genetically or by the application of known defense-inducing
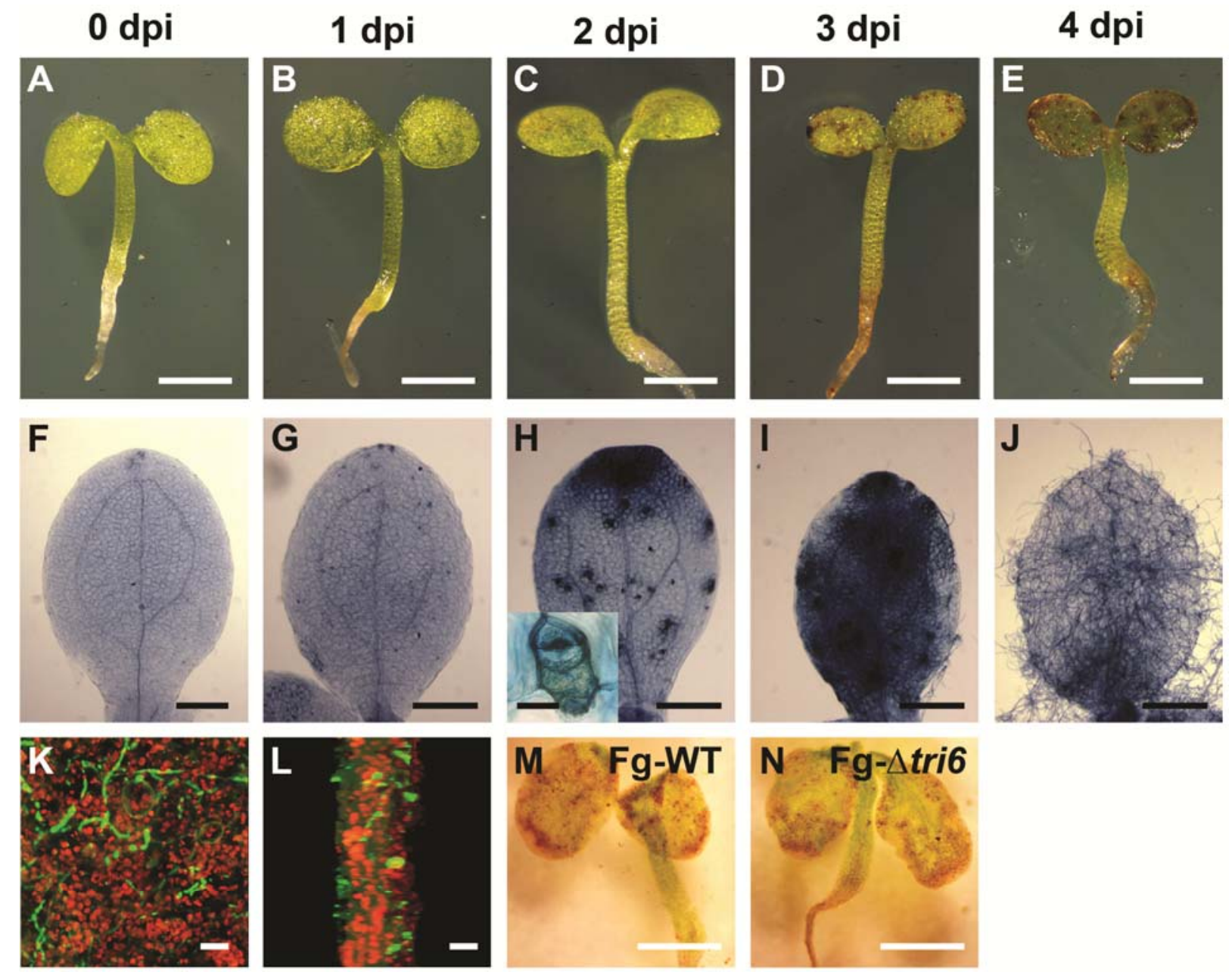

Fig. 1. Phenotype of Arabidopsis seedlings inoculated with Fusarium graminearum in liquid media. A to E, Macroscopic symptom development in infected seedlings. Timescale is given in days postinoculation (dpi). Scale bars indicate $1 \mathrm{~mm}$. F to J, Microscopic phenotypes observed following trypan blue staining. Punctate staining at early time points is generally centered on stomata (inset in $\mathrm{H}$ ), while proliferating fungal mycelia are also stained at later time points. Scale bars indicate $0.25 \mathrm{~mm}(20 \mu \mathrm{m}$ for inset). $\mathbf{K}$ and $\mathbf{L}$, Growth of green fluorescent protein-labeled F. graminearum in Arabidopsis cotyledons at 4 dpi. Images represent an overhead view of adaxial mesophyll cells $(\mathrm{K})$ and a side view reconstructed from multiple $\mathrm{z}$-stacks $(\mathrm{L})$. Scale bars indicate $10 \mu \mathrm{m}$. $\mathbf{M}$ and $\mathbf{N}$, Influence of deoxynivalenol (DON) production on symptom development in Arabidopsis. Wells were inoculated with either wild-type $F$. graminearum (Fg-WT) (M) or a $\Delta$ tri6 mutant incapable of producing DON (Fg- $\Delta$ tri6) $(\mathrm{N})$. Images were captured at 4 dpi. Scale bars indicate $1 \mathrm{~mm}$. All images are representative of observations from at least three biological replications. 
molecules. We observed that overexpression of a GOLDEN2LIKE (GLKI) gene dramatically reduced fungal infection of Arabidopsis seedlings (Fig. 2B), as shown previously using soil-grown plants (Savitch et al. 2007). Similarly, disruption of jasmonic acid (JA) signaling in coronatine insensitive 1 (coil) and jasmonate resistant 1 (jarl) mutants was associated with minimal symptom development following inoculation with $F$. graminearum (Fig. 2C and D), also in line with previous ob- servations (Makandar et al. 2010). Exogenous application of the basal defense-eliciting peptide flg22 prior to inoculation also protected seedlings from infection, and this protection was eliminated in seedlings lacking the flg22 receptor FLAGELLIN-SENSITIVE 2 (FLS2) (Fig. 2G and H). Finally, when seedlings were pretreated with the antifungal compound hygromycin, all plant tissues remained relatively green and mycelial growth in the wells was negligible (Fig. 2F). Overall, the phe-
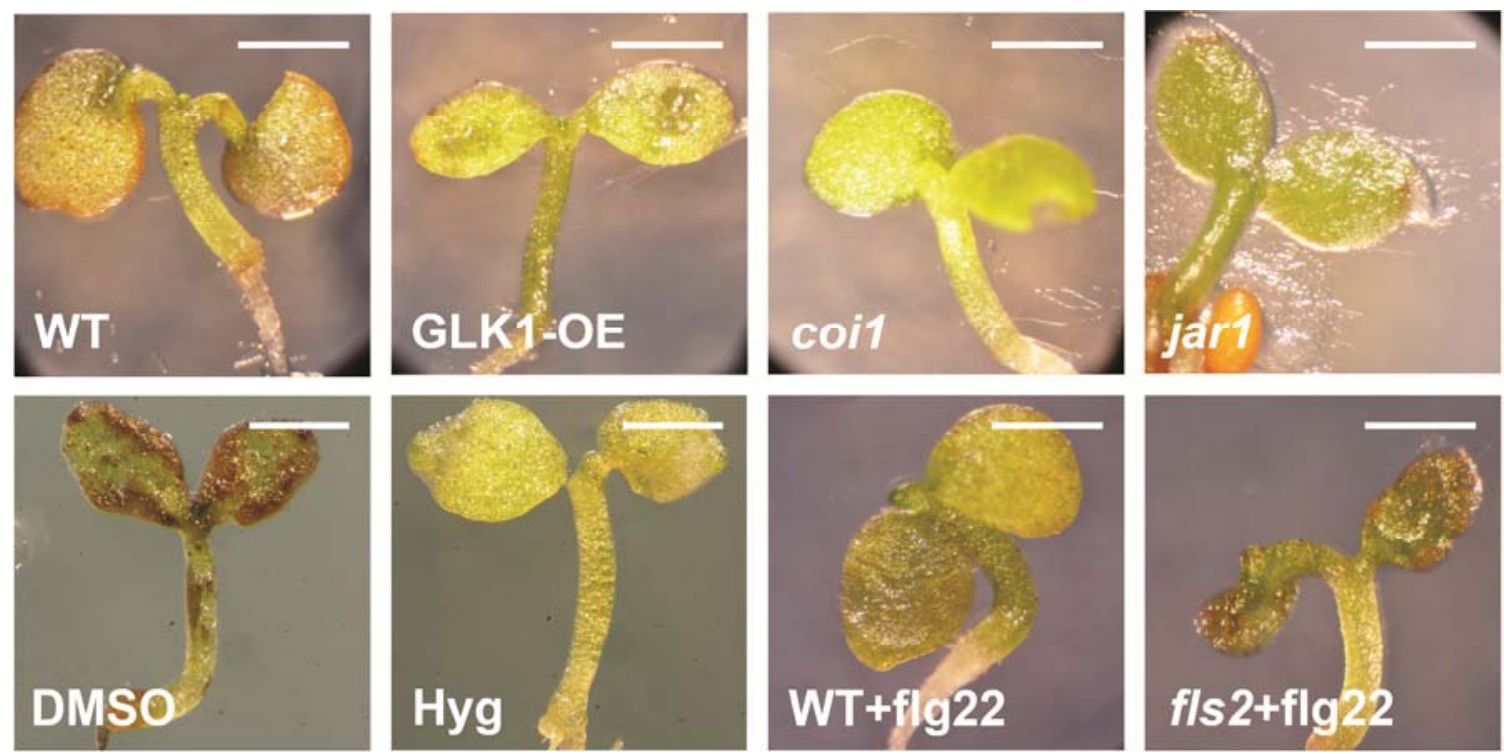

Fig. 2. The Arabidopsis-Fusarium graminearum interaction can be attenuated by exogenous application of small molecules or by genetically altering host defense responses. In addition to wild-type Arabidopsis (Col-0) (WT), a line overexpressing GLK1 (GLK1-OE) was examined, as well as coil and jarl mutants. WT seedlings were also treated with $1 \%$ dimethyl sulfoxide (DMSO), $25 \mu \mathrm{M}$ hygromycin (Hyg), or $1 \mu \mathrm{M}$ flg22 peptide (WT+flg22) $12 \mathrm{~h}$ prior to inoculation. The flg22 peptide was also tested on $f l s 2$ mutants $(f l s 2+\mathrm{flg} 22)$. Scale bars indicate $1 \mathrm{~mm}$. All images are representative of observations from at least three biological replications.
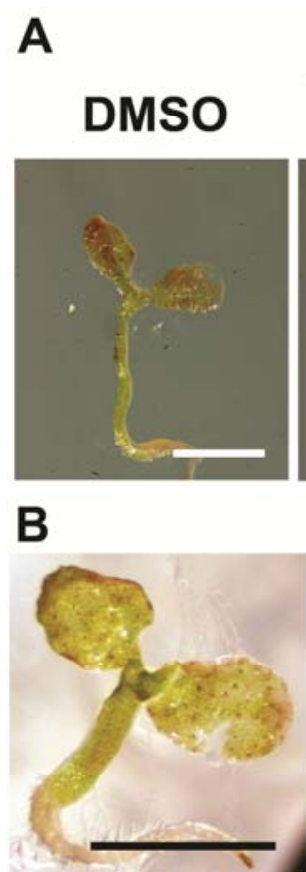

DMSO
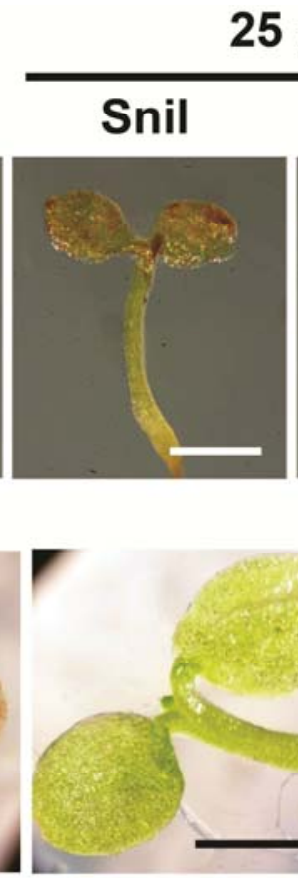

$\mu \mathrm{M}$

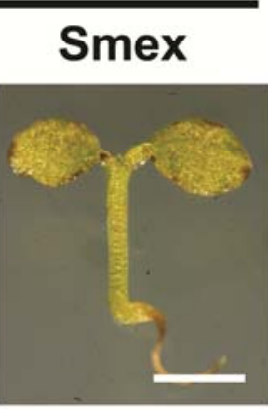

C<smiles>Nc1ccc(S(N)(=O)=O)cc1</smiles>

Snil $\mathbf{5 0} \mu \mathbf{M}$

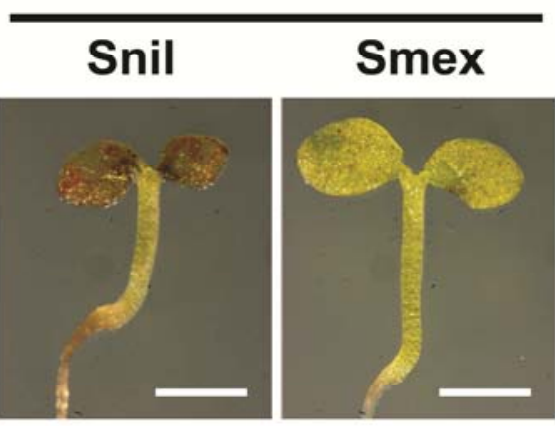

\section{$50 \mu \mathbf{M}$ \\ Gramine}

Fig. 3. Identification of novel small molecules that protect Arabidopsis seedlings from infection by Fusarium graminearum. A, Differential activity of sulfamethoxazole (Smex) and sulfanilamide (Snil) in the liquid assay. DMSO = dimethyl sulfoxide. B, Protection of Arabidopsis seedlings from F. graminearum infection by $50 \mu \mathrm{M}$ gramine, a compound identified from a collection of natural products. C, Chemical structures of Smex, Snil, and gramine. All images are representative of observations from at least three biological replications. 
notypes of the resistant host genotypes and small moleculetreated seedlings were very consistent across both technical and biological replicates. These results indicated that infection of Arabidopsis seedlings in liquid media could be affected by altering host defenses or pathogen growth.

\section{Chemical screening.}

Based on the findings summarized in Figure 2, we conducted a small-scale screen to identify novel compounds capable of preventing the infection of Arabidopsis seedlings by $F$. graminearum. In a prior screen, we identified a family of sulfanilamide-based compounds that protected Arabidopsis from infection by $P$. syringae (Schreiber et al. 2008). Sulfamethoxazole (Smex) exhibited the strongest activity, while the sulfanilamide (Snil) core group alone was essentially inactive in the liquid assay (Schreiber et al. 2008). Remarkably, these observations were recapitulated with $F$. graminearum (Fig. 3A). Very little spotting or browning was observed in seedlings pretreated with $25 \mu \mathrm{M}$ Smex and, at $50 \mu \mathrm{M}$, seedlings were virtually symptom free. Seedlings pretreated with Snil, however, were indistinguishable from dimethyl sulfoxide (DMSO)treated controls. We also screened 80 chemicals from a natural product collection and identified the indole alkaloid gramine (also called donaxine) as having strong protective activity (Fig. 3B). Interestingly, this compound did not protect seedlings from infection by $P$. syringae (data not shown).

\section{Experimental translation from Arabidopsis to wheat.}

We next investigated whether chemicals identified through an Arabidopsis-based screen would exhibit similar activity on wheat, a natural host of $F$. graminearum. Two chemical treatment regimes were employed, involving either simultaneous application of fungal spores and chemical to a single spikelet ("antagonism") or chemical pretreatment of one spikelet followed by inoculation of an adjacent spikelet $24 \mathrm{~h}$ later ("priming"). We subsequently monitored the development of macroscopic disease symptoms over time and measured the accumulation of DON in wheat heads at 21 days postinfection. For both methods, inoculated heads pre- or co-treated with DMSO exhibited extensive discoloration that eventually affected nearly $100 \%$ of spikelets (Fig. 4A; Supplementary Table 1). This phenotype coincided with DON accumulations in excess of $600 \mathrm{ppm}$. Co-application of $50 \mu \mathrm{M}$ Smex and F. graminearum significantly reduced the infection to $43 \%$ of spikelets and lowered DON concentrations to $20 \%$ of that measured in the DMSO control. These reductions were less dramatic for Snil, and only the effect on DON accumulation was statistically significant. Gramine also exhibited weak activity and, again, significant reductions were observed only for DON content $(42 \%$ of control). Priming experiments corroborated the strong activity of Smex, which reduced spikelet infection by over $50 \%$ and DON accumulation by $75 \%$. Snil did not have a significant impact on either measurement. Priming with gramine, however, resulted in the infection of $30 \%$ fewer spikelets and close to $75 \%$ less DON accumulation. Neither Smex nor gramine significantly inhibited fungal growth in vitro at a concentration of $50 \mu \mathrm{M}$ (Fig. 4B), suggesting that these compounds induce defense responses in wheat that hinder $F$. graminearum infection. Overall, these results demonstrate that the Arabidopsis-F. graminearum pathosystem can be used to identify disease control strategies in wheat.

In addition to directly treating or inoculating single spikelets, we also evaluated the activity of gramine and Smex by spraying the chemicals on wheat heads followed by spray inoculation $24 \mathrm{~h}$ later. When applied at a concentration of 50 $\mu \mathrm{M}$, neither gramine nor Smex significantly altered the development of $F$. graminearum infection symptoms (data not shown). Considering that spray treatment likely lowers the effective dose of chemical that reaches floral tissues, we tested higher concentrations of each compound along with a lower inoculum concentration, as used by Gilbert and associates (2001). With this method, only a subset of inoculated heads actually became infected, even after several weeks (Fig. 5). Notably, however, pretreatment of heads with $250 \mu \mathrm{M}$ gramine or Smex reduced the number of infected heads by approximately $50 \%$ relative to the DMSO control (Fig. 5; Supplementary Table 2). The spread of disease within these infected heads was also significantly reduced by at least $30 \%$ (Fig. 5; Supplementary Table 3 ). These results provide a promising indication that both gramine and Smex retain their activity under "realworld" conditions.

\section{DISCUSSION}

As a small, well-characterized model plant, Arabidopsis has tremendous potential utility as a surrogate for studies of fungal pathology in more genetically complex plant hosts. We examined the interaction between Arabidopsis and $F$. graminearum in a 96-well assay format as a precursor to more high-throughput analyses. The inoculation of Arabidopsis seedlings in liquid media with $F$. graminearum macroconidia resulted in the appearance of dark, lesion-like spots on the cotyledons of infected seedlings. Later stages of infection were characterized by extensive spotting and significant loss of tissue integrity. Such apparent necrosis was previously observed after inoculation of wounded siliques (Cuzick et al. 2008) and spray inoculation of floral organs (Urban et al. 2002). Interestingly, similar infection symptoms were observed on detached leaves only when these tissues were wounded prior to inoculation (Chen et al. 2006). In our liquid assay, the proliferation of $F$. graminearum in intact Arabidopsis cotyledons is likely aided by the continuous contact between host and pathogen under maximally humid conditions, which favor fungal growth. Cotyledons may also be more susceptible to infection than mature Arabidopsis tissues (Aarts et al. 1998).

At the microscopic level, trypan blue staining identified areas of cell death that increased in both size and number over time. Notably, staining was initially localized to guard cells and some neighboring cells, in agreement with observations by Cuzick and associates (2008). This also provides an important correlate to wheat, in which stomata are key points of entry for $F$. graminearum (Pritsch et al. 2000). Indeed, Desmond and associates (2008) noted that inoculation of wheat leaf tissue with $F$. graminearum induced stomatal $\mathrm{H}_{2} \mathrm{O}_{2}$ accumulation and eventual guard cell death at the site of infection. The extent to which these events reflect induced host defense responses versus consequences of necrotrophic pathogenesis remains to be investigated.

One aspect of $F$. graminearum pathogenesis that appears to differ between Arabidopsis and wheat is the virulence contribution of the mycotoxin DON. Strains of $F$. graminearum lacking DON are significantly less capable of colonizing and spreading within wheat tissues, although virulence on other cereal crops may be unaffected (Jansen et al. 2005; Maier et al. 2006; Proctor et al. 1995). We noted that the absence of DON did not alter the infection phenotype of Arabidopsis seedlings in liquid media, similar to previous observations from silique inoculation assays (Cuzick et al. 2008). As such, the liquid assay provides the opportunity to probe mechanisms of disease resistance in a DON-independent manner.

Having noted a salient, reproducible infection phenotype in the liquid assay, we then sought to survey factors that may influence the outcome of the Arabidopsis- $F$. graminearum interaction. Overexpression of $G L K 1$, a transcriptional activator, was 


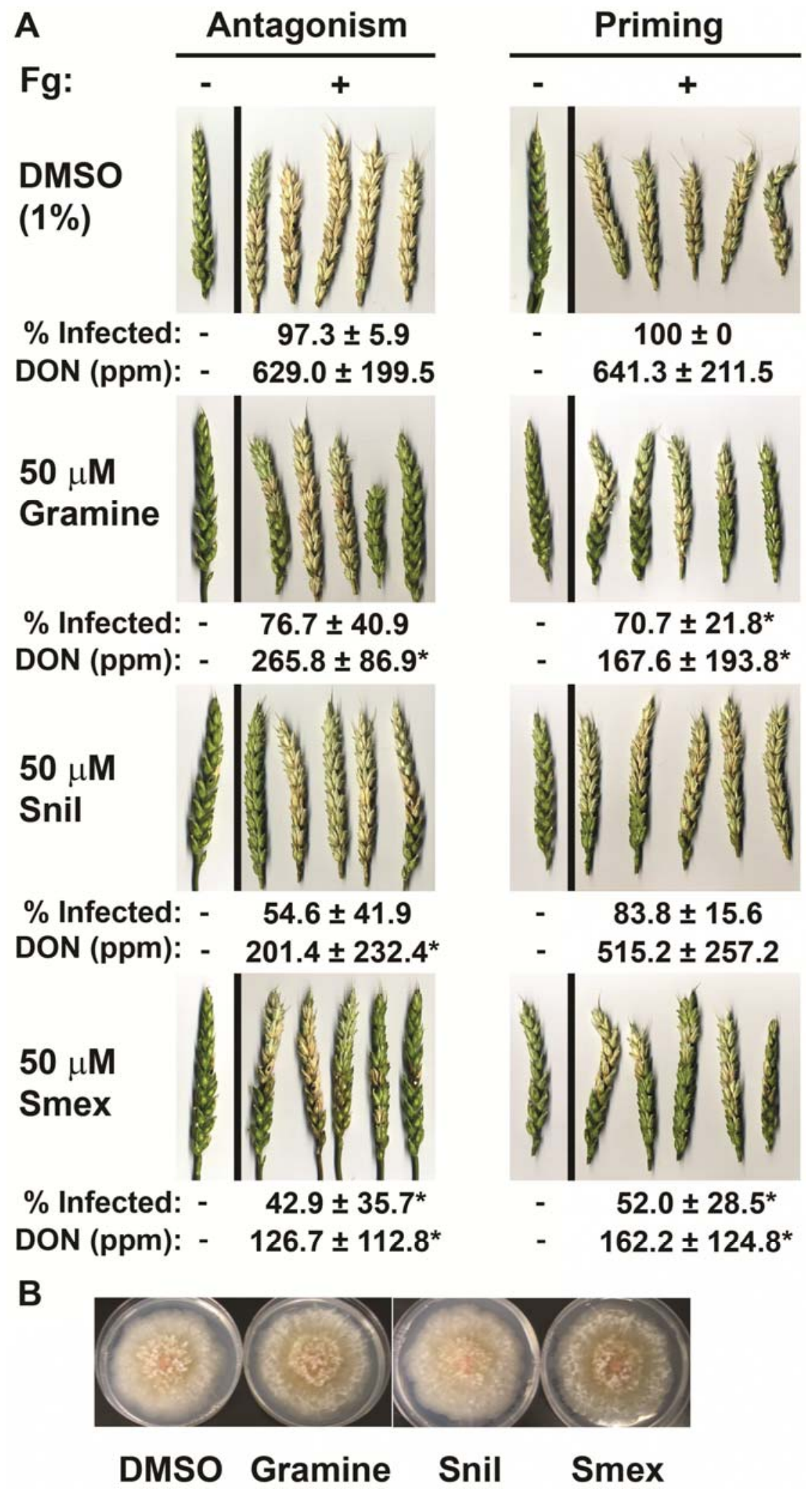

Fig. 4. Activity of gramine and sulfamethoxazole in the wheat-Fusarium graminearum pathosystem as evaluated using single spikelet treatment and inoculation approaches. A, A single spikelet within each wheat head was either simultaneously treated with chemical and F. graminearum (Fg) conidiospores ("antagonism") or locally pretreated with chemical at a single spikelet, then inoculated at an adjacent spikelet $24 \mathrm{~h}$ later ("priming"). Images were captured at 21 days postinoculation (dpi) for co-application experiments and $14 \mathrm{dpi}$ for priming experiments. Average percent spikelet infection per head (\% Infection) is indicated \pm standard deviation. Mean deoxynivalenol (DON) concentrations were also measured in wheat heads at the above time points and are provided in parts per million $(\mathrm{ppm}) \pm$ standard deviation. In uninoculated heads, the baseline DON measurement is invariably 0.8 ppm. Asterisks indicate a statistically significant difference between the treatment and the dimethyl sulfoxide (DMSO) control in three independent experiments, as determined by a Student's $t$ test $(\alpha=0.05)$. Abbreviations: $-=$ uninoculated, $+=$ inoculated, Smex $=$ sulfamethoxazole, Snil $=$ sulfanilamide. B, Growth of $F$. graminearum on potato dextrose agar media supplemented with $50 \mu \mathrm{M}$ Smex, Snil, or gramine. Control plates contain $1 \%$ DMSO. Images are representative of observations from at least three biological replications. 
previously shown to enhance the resistance of Arabidopsis to infection by $F$. graminearum (Savitch et al. 2007). Similar enhancements were demonstrated in coil and jarl mutants (Makandar et al. 2010). Notably, all three of these genotypes were originally assessed by pressure infiltration of leaves from soil-grown adult plants. Our corroboration of the resistance phenotypes in seedlings grown in liquid media thus validates the biological relevance of this Arabidopsis assay.

There are, however, some notable differences in resistance mechanisms against $F$. graminearum between Arabidopsis and wheat. Prior observations from the liquid assay indicated that the bacterial flagellin peptide flg22 could protect Arabidopsis seedlings from infection by P. syringae (Schreiber et al. 2008). We demonstrated similar activity for this peptide against $F$. graminearum, including a dependence on the FLS2 receptor (Fig. 2). A wide range of defense responses are induced by pathogen-associated molecular patterns such as flg22, including callose deposition in cell walls and expression of defense genes (Gómez-Gómez et al. 1999; Jones and Dangl 2006; Pitzschke et al. 2009; Schwessinger and Zipfel 2008). Our results indicate that the elicitation of this basal immune response is an effective strategy for protecting Arabidopsis from both bacterial and fungal pathogens. However, we were unable to detect significant flg22-induced resistance in wheat (data not shown), suggesting that any responses induced by this peptide are relatively weak and that other pathogen-associated molecular patterns may play a more prominent role in the elicitation of wheat defenses (data not shown). Furthermore, although JA appears to play a negative role in Arabidopsis resistance against $F$. graminearum, expression studies suggest that JA may play a positive role in the resistance of wheat against $F$. graminearum and $F$. pseudograminearum (Desmond et al. 2006; Li and Yen 2008). Despite these differences, we have demonstrated that Arabidopsis can be used to identify small molecules that can protect wheat against $F$. graminearum infection.

We also observed effective control of $F$. graminearum infection in Arabidopsis by the antifungal compound hygromycin. In addition to the absence of infection symptoms on seedlings, very few mycelia were present in the liquid media, contrasting with the profuse mycelial growth in control wells and those containing the resistant Arabidopsis genotypes (data not shown). This illustrates one of the strengths of the liquid assay, in that the simultaneous evaluation of both host and pathogen allows rapid characterization of chemicals in terms of antifungal activity, efficacy of plant protection, and potential phytotoxicity all in the same well.

Further to these advantages, and perhaps most importantly, we have demonstrated that novel compounds identified using an Arabidopsis-based liquid assay exhibit similar protective activity on wheat. The strongest activity was displayed by Smex, a compound also shown to protect Arabidopsis from infection by $P$. syringae (Schreiber et al. 2008). In the wheat$F$. graminearum interaction, the retention of significant activity in the priming experiment suggests that some type of plant defense response is being activated by this compound. It remains to be determined whether Smex-induced resistance against Fusarium spp. employs a salicylic acid- and NPR1independent pathway as it does against $P$. syringae (Schreiber et al. 2008). At the same time, Snil significantly reduced the extent of infection only when co-applied with the fungus, indicating that there may be a direct effect on some aspect of fungal virulence that relies solely on the Snil core group.

The indole alkaloid gramine was also found to reduce the severity of $F$. graminearum infection in wheat. Although weaker than Smex, gramine reduced DON accumulation in both antagonism and priming experiments. These results suggest that both gramine and Smex influence type II or type III resistance against $F$. graminearum. The spray experiments also indicate that type I resistance is affected. Such outcomes could arise from the induction of host defenses or the inhibition of some aspect of fungal virulence. Gramine has long been recognized in barley (Hordeum vulgare L.) as a root-secreted allelochemical (Overland 1966), but it is also present in leaves and further accumulates in response to abiotic, biotic, and mechanical stresses (Hanson et al. 1983; Hautala and Holopainen 1995; Matsuo et al. 2001). In addition, exogenously applied gramine was shown to reduce aphid feeding and fecundity on barley (Corcuera 1993; Zúñiga et al. 1988) and inhibit spore germination by the powdery mildew fungus Erysiphe graminis

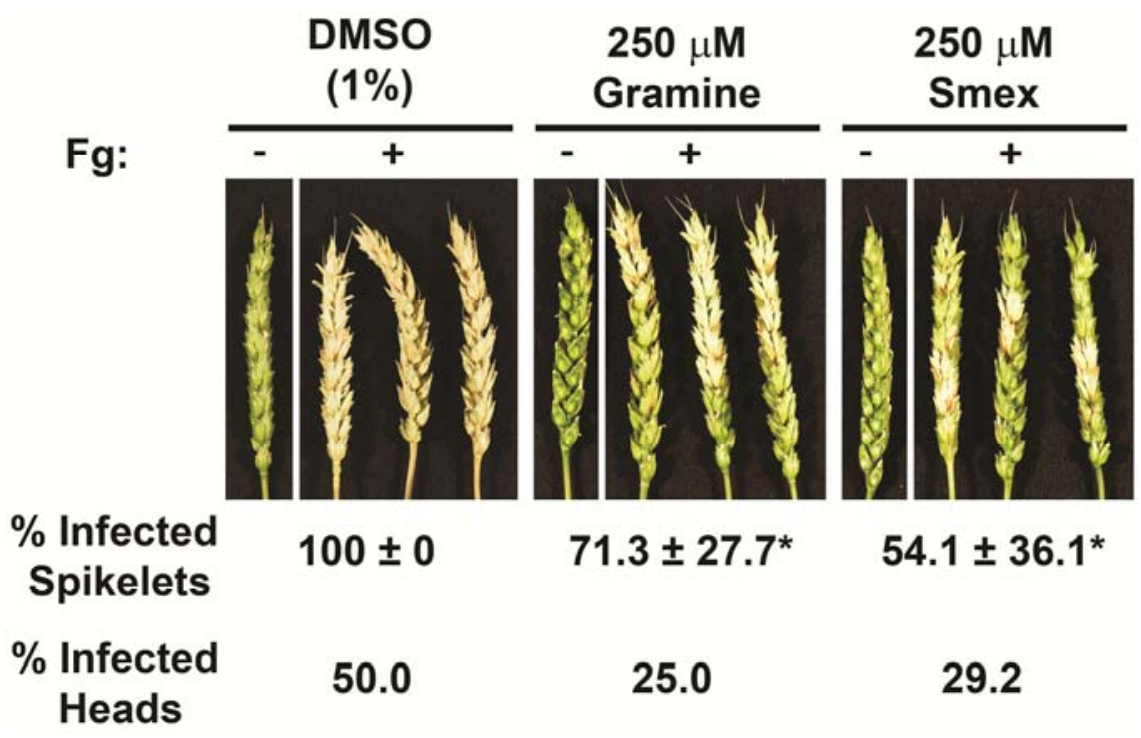

Fig. 5. Activity of gramine and sulfamethoxazole (Smex) in the wheat-Fusarium graminearum pathosystem as evaluated using a spray treatment and inoculation approach. Wheat heads were sprayed with chemicals, then sprayed with an F. graminearum (Fg) spore suspension 24 h later. At 28 days postinoculation, the number of wheat heads exhibiting symptoms of $F$. graminearum infection was recorded (\% Infected Heads). Representative images of infected spikelets are shown. Average percent spikelet infection within the infected heads (\% Infected Spikelets) is indicated \pm standard deviation. Asterisks indicate a statistically significant difference between the treatment and the dimethyl sulfoxide (DMSO) control in two independent experiments, as determined by a Student's $t$ test $(\alpha=0.05)$. Abbreviations: $-=$ uninoculated and $+=$ inoculated. 
f. sp. hordei (Wippich and Wink 1985). Investigations of gramine toxicity indicate that this compound uncouples electron transport in plant and cyanobacterial thylakoids (Andreo et al. 1984; Foguel and Chaloub 1993) as well as in mammalian mitochondria (Niemeyer and Roveri 1984). The mechanism through which gramine reduces susceptibility to $F$. graminearum infection remains to be determined.

In summary, we have described an assay for high-throughput investigations of the Arabidopsis-F. graminearum pathosystem. Of major significance is our demonstration that information obtained from the genetically tractable Arabidopsis system can be extended to an economically important crop species. The ability to translate screening results not only from a dicot to a monocot but also from plants at different developmental stages grown in vastly different environments strongly reinforces the biological relevance of the liquid assay. This also suggests that the liquid assay could potentially be used to study other agriculturally relevant pathogens. Furthermore, our screening and subsequent translation experiments were performed with compounds at micromolar concentrations, whereas most commercial fungicides currently registered for use against Fusarium spp. are applied at millimolar concentrations. As such, the liquid assay provides a relatively stringent environment conducive to the identification of chemicals with strong activity in the host or pathogen. Given recent observations of $F$. graminearum isolates with resistance to multiple fungicides (Becher et al. 2010; Chen and Zhou 2009), the identification of crop protectants with alternative modes of action is an increasingly urgent task. This challenge can be addressed through the development of novel high-throughput assays, as we have demonstrated with the Arabidopsis- $F$. graminearum liquid pathosystem.

\section{MATERIALS AND METHODS}

\section{Fungal strains and inoculum preparation.}

F. graminearum Schwabe (teleomorph G. zeae (Schwein.) Petch) wild-type strain DAOM 233423 was obtained from the Canadian Collection of Fungal Cultures, Agriculture and AgriFood Canada, Ottawa, Canada. A $\Delta$ tri6 strain was generated by homologous recombination (Supplementary Fig. 1). The $F$. graminearum-GFP (Fg-GFP) strain was obtained from R. Proctor, National Center for Agricultural Utilization Research, Agricultural Research Service, United States Department of Agriculture, Peoria, IL U.S.A. Fungi were grown on potato dextrose agar (PDA) media for routine culture and analyses. To prepare conidiospores for pathogenicity assays, an approximately 3-mm plug was extracted from a fresh PDA plate culture and transferred to $100 \mathrm{ml}$ of CMC media (Cappellini and Peterson 1965). Cultures were shaken at $28^{\circ} \mathrm{C}$ for 3 to 5 days to generate conidia. Mycelial solids were separated from conidia by passing through four layers of sterile cheesecloth. Conidia were then washed with sterile water twice by centrifugation at 4,000 rpm for $15 \mathrm{~min}$ at room temperature. The lightly pelleted conidia were resuspended in sterile water and then stored at $4^{\circ} \mathrm{C}$. Conidia were inspected and counted with a hemacytometer prior to use.

\section{Arabidopsis-Fusarium graminearum 96-well plate pathogenicity assay.}

Arabidopsis thaliana (ecotype Columbia) was used for liquid assays, which also included coil and jarl mutants, obtained from J. Dangl, University of North Carolina. A homozygous TDNA insertion line (SALK_026801C) affecting the FLAGELLIN-SENSITIVE 2 (FLS2) gene (At5g46330) was obtained from the Arabidopsis Biological Resource Center. Assays in liquid media were conducted essentially as outlined in Schreiber and associates (2008). Briefly, 5 to 10 Arabidopsis seeds were distributed into each well of 96-well plates, with the exception of the 36 most peripheral wells. Plates with seed were sterilized by exposure to chlorine gas, and $200 \mu$ l of liquid media $(0.5 \times$ Murashige and Skoog basal media [Sigma-Aldrich, Oakville, ON, Canada] and $2.5 \mathrm{mM}$ 2-[N-morpholino]ethanesulfonic acid, pH 5.8 [Sigma-Aldrich]) was added to each well. After 4 days of stratification at $4^{\circ} \mathrm{C}$, covered plates were placed under continuous light at $22^{\circ} \mathrm{C}$. Five-day-old seedlings were inoculated with $F$. graminearum conidiospore suspensions at a final concentration of 50 to 500 spores $/ \mathrm{ml}$. Covered plates were gently agitated on a Heidolph Titramax 1000 vibrating shaker (VWR, Mississauga, ON, Canada) at $600 \mathrm{rpm}$ under continuous light at $22^{\circ} \mathrm{C}$ in a controlled environment chamber. Seedling phenotypes were assessed at 4 dpi. Images of macroscopic disease symptoms were collected by bright-field microscopy of whole seedlings mounted on $1.5 \%$ agar plates. Plant cell death and fungal growth within seedlings were both visualized by lactophenol-trypan blue staining (Koch and Slusarenko 1990). Samples were viewed with a Leica MZFLIII stereomicroscope (Leica Microsystems, Wetzlar, Germany) and all photographs taken with either a Canon EOSD60 digital camera (Canon Inc., Tokyo) or a MicroPublisher 3.3 RTV camera (QImaging, Surrey, BC, Canada). For confocal imaging, seedlings were mounted on slides and viewed with a Zeiss LSM 510 META confocal microscope (Carl Zeiss Canada Ltd., Toronto). GFPlabeled mycelia were visualized using an excitation wavelength of $488 \mathrm{~nm}$ and a 500- to 530-nm emission filter. All confocal images were processed with the Zeiss LSM image browser. Images were assembled in Adobe Photoshop CS2 (Adobe Systems, Mountain View, CA, U.S.A.).

\section{Chemical screening.}

The TimTec NP280 natural product collection (TimTec LLC, Newark, DE, U.S.A.) was provided as $2.5 \mathrm{mM}$ stock solutions dissolved in DMSO. One plate of these chemicals (80 compounds) was screened using 5-day-old seedlings grown in liquid media as described above. Compounds were added to each well at a final concentration of $25 \mu \mathrm{M}$. Each plate included a well containing $25 \mu \mathrm{M}$ hygromycin as a positive control, along with several negative control wells containing $1 \%$ DMSO. Plates were stored in the dark at room temperature for $12 \mathrm{~h}$, then inoculated with $F$. graminearum and incubated as described above. Seedling phenotypes were assessed at 3 to 4 dpi. All compounds were screened in triplicate. Experiments using flg22 peptide (Sheldon Biotechnology Centre, Montreal) as well as retests of individual compounds (obtained from Sigma-Aldrich) were conducted in the same manner.

\section{Wheat cultivation and pathogenicity assays.}

All wheat pathogenicity assays were performed on Triticum aestivum L. 'Roblin', which is susceptible to F. graminearum. Seed were provided by André Kalikilio, Agriculture and AgriFood Canada, Ottawa. Seed were surface sterilized with 50\% sodium hypochlorite and washed three times with sterile distilled water. Seed were then transferred to $1 \%$ water agar plates supplemented with streptomycin at $50 \mu \mathrm{g} / \mathrm{ml}$. After $48 \mathrm{~h}$, germlings were planted out (two per pot) in 4-in. fiber pots. Planting medium consisted of three parts topsoil, two parts peat moss, and one part sand, plus dolomitic lime for $\mathrm{pH}$ adjustment. Potted seedlings were cultivated in a growth chamber under both fluorescent and incandescent lighting to provide a 16-h photoperiod and a temperature cycle of 22 and $15^{\circ} \mathrm{C}$, day and night, respectively. Soil was amended with 20-20-20 fertilizer twice weekly for the first 4 weeks of growth, then once weekly until mid-anthesis (approximately 6 to 7 weeks), at which point pathogenicity assays were performed. 
Two different methods were used to evaluate the activity of chemicals in wheat pathogenicity assays. Inocula for "antagonism" experiments contained $50 \mu \mathrm{M}$ of the chemical compound and 1,000 F. graminearum conidiospores in a 10- $\mu \mathrm{l}$ volume. This solution was applied interior to the lemma of one spikelet midway down the head, using 5 to 10 heads per treatment. A spore suspension containing 1\% DMSO was used as a control. For "priming" experiments, chemicals $(50 \mu \mathrm{M})$ in a $10-\mu l$ volume were applied to one spikelet per head, followed by inoculation of an adjacent spikelet $24 \mathrm{~h}$ later with a $10-\mu \mathrm{l}$ droplet containing 1,000 spores. In a variation of the priming experiment, wheat heads were sprayed with a solution of 250 $\mu \mathrm{M}$ chemical plus $0.01 \%$ Silwet L-77 surfactant (GE Silicones, South Charleston, WV, U.S.A.), then sprayed $24 \mathrm{~h}$ later with a suspension of $5 \times 10^{4}$ spores/ml plus $0.01 \%$ Silwet. For all evaluation methods, inoculated plants were transferred to a spray facility under greenhouse conditions. Plants were monitored for the development of disease symptoms such as spikelet discoloration and analyzed when heads in the control treatment group exhibited approximately $100 \%$ spikelet infection. The concentration of DON in inoculated tissues was measured as previously described (Sinha et al. 1995).

\section{ACKNOWLEDGMENTS}

K. J. Schreiber and C. G. Nasmith contributed equally to this manuscript. K. Schreiber was supported by the Natural Science and Engineering Research Council (NSERC) of Canada. C. G. Nasmith was funded by a grant from the Grain Farmers of Ontario. D. Desveaux is a Canada Research Chair in Plant-Microbe Systems Biology. This work was supported by an NSERC Discovery Grant and awards from the Canadian Foundation for Innovation and the Ontario Ministry of Research and Innovation to D. Desveaux. We thank J. Dangl for Arabidopsis mutant lines, R. Proctor for the $F$. graminearum-GFP strain, D. Chabot for technical assistance with confocal microscopy, and the handling editor and anonymous reviewers for their insightful comments.

\section{LITERATURE CITED}

Aarts, N., Metz, M., Holub, E., Staskawicz, B. J., Daniels, M. J., and Parker, J. E. 1998. Different requirements for EDS1 and NDRl by disease resistance genes define at least two $R$ gene-mediated signaling pathways in Arabidopsis. Proc. Natl. Acad. Sci. U.S.A. 95:10306-10311.

Anderson, J. A., Stack, R. W., Liu, S., Waldron, B. L., Fjeld, A. D., Coyne, C., Moreno-Sevilla, B., Fetch, J. M., Song, Q. J., Cregan, P. B., and Frohberg, R. C. 2001. DNA markers for Fusarium head blight resistance QTLs its two wheat populations. Theor. Appl. Genet. 102:1164-1168.

Andreo, C. S., Orellano, E. G., and Niemeyer, H. M. 1984. Uncoupling of spinach thylakoids by gramine. Z. Naturforsch. C 39:746-748.

Bai, G. H., and Shaner, G. 2004. Management and resistance in wheat and barley to Fusarium head blight. Annu. Rev. Phytopathol. 42:135-161.

Ban, T., and Suenaga, K. 2000. Genetic analysis of resistance to Fusarium head blight caused by Fusarium graminearum in Chinese wheat cultivar Sumai 3 and the Japanese cultivar Saikai 165. Euphytica 113:87-99.

Becher, R., Hettwer, U., Karlovsky, P., Deising, H. B., and Wirsel, S. G. R. 2010. Adaptation of Fusarium graminearum to tebuconazole yielded descendants diverging for levels of fitness, fungicide resistance, virulence, and mycotoxin production. Phytopathology 100:444-453.

Cappellini, R. A., and Peterson, J. L. 1965. Macroconidium formation in submerged cultures by a nonsporulating strain of Gibberella zeae. Mycologia 57:962-966.

Chen, X. W., Steed, A., Harden, C., and Nicholson, P. 2006. Characterization of Arabidopsis thaliana-Fusarium graminearum interactions and identification of variation in resistance among ecotypes. Mol. Plant Pathol. 7:391-403.

Chen, Y., and Zhou, M. G. 2009. Characterization of Fusarium graminearum isolates resistant to both carbendazim and a new fungicide JS39919. Phytopathology 99:441-446.

Corcuera, L. J. 1993. Biochemical basis for the resistance of barley to aphids. Phytochemistry 33:741-747.

Cundliffe, E., Cannon, M., and Davies, J. 1974. Mechanism of inhibition of eukaryotic protein synthesis by trichothecene fungal toxins. Proc. Natl. Acad. Sci. U.S.A. 71:30-34.

Cuthbert, P. A., Somers, D. J., Thomas, J., Cloutier, S., and Brûlé-Babel,
A. 2006. Fine mapping Fhb1, a major gene controlling Fusarium head blight resistance in bread wheat (Triticum aestivum L.). Theor. Appl. Genet. 112:1465-1472.

Cuzick, A., Urban, M., and Hammond-Kosack, K. 2008. Fusarium graminearum gene deletion mutants mapl and tri5 reveal similarities and differences in the pathogenicity requirements to cause disease on Arabidopsis and wheat floral tissue. New Phytol. 177:990-1000.

Desmond, O. J., Edgar, C. I., Manners, J. M., Maclean, D. J., Schenk, P. M., and Kazan, K. 2006. Methyl jasmonate induced gene expression in wheat delays symptom development by the crown rot pathogen Fusarium pseudograminearum. Physiol. Mol. Plant. Pathol. 67:171-179.

Desmond, O. J., Manners, J. M., Stephens, A. E., Maclean, D. J., Schenk, P. M., Gardiner, D. M., Munn, A. L., and Kazan, K. 2008. The Fusarium mycotoxin deoxynivalenol elicits hydrogen peroxide production, programmed cell death and defence responses in wheat. Mol. Plant Pathol. 9:435-445.

Foguel, D., and Chaloub, R. M. 1993. Effects of the alkaloid gramine on the light-harvesting, energy transfer, and growth of Anabaena sp. (PCC7119). Plant Physiol. 101:633-639.

Gilbert, J., Abramson, D., McCallum, B., and Clear, R. 2001. Comparison of Canadian Fusarium graminearum isolates for aggressiveness, vegetative compatibility, and production of ergosterol and mycotoxins. Mycopathologia 153:209-215.

Gómez-Gómez, L., Felix, G., and Boller, T. 1999. A single locus determines sensitivity to bacterial flagellin in Arabidopsis thaliana. Plant J. 18:277-284.

Goswami, R. S., and Kistler, H. C. 2004. Heading for disaster: Fusarium graminearum on cereal crops Mol. Plant Pathol. 5:515-525.

Hanson, A. D., Ditz, K. M., Singletary, G. W., and Leland, T. J. 1983. Gramine accumulation in leaves of barley grown under high-temperature stress. Plant Physiol. 71:896-904.

Hautala, E. L., and Holopainen, J. K. 1995. Gramine and free amino acids as indicators of fluoride-induced stress in barley and its consequences to insect herbivory. Ecotox. Environ. Safe. 31:238-245.

Hohn, T. M., McCormick, S. P., Alexander, N. J., Desjardins, A. E., and Proctor, R. H. 1998. Function and biosynthesis of trichothecenes produced by Fusarium species. Pages 17-24 in: Molecular Genetics of Host-Specific Toxins in Plant Disease. K. Kohmoto and O.C. Yoder, eds. Kluwer Academic Publishers, Boston.

Illergård, K., Ardell, D. H., and Elofison, A. 2009. Structure is three to ten times more conserved than sequence: A study of structural response in protein cores. Proteins Struct. Funct. Bioinf. 77:499-508.

Jansen, C., von Wettstein, D., Schäfer, W., Kogel, K. H., Felk, A., and Maier, F. J. 2005. Infection patterns in barley and wheat spikes inoculated with wild-type and trichodiene synthase gene disrupted Fusarium graminearum. Proc. Natl. Acad. Sci. U.S.A. 102:16892-16897.

Jones, J. D. G., and Dangl, J. L. 2006. The plant immune system. Nature 444:323-329.

Kawasumi, M., and Nghiem, P. 2007. Chemical genetics: Elucidating biological systems with small-molecule compounds. J. Invest. Dermatol. 127:1577-1584.

Koch, E., and Slusarenko, A. 1990. Arabidopsis is susceptible to infection by a downy mildew fungus. Plant Cell 2:437-445.

Li, G., and Yen, Y. 2008. Jasmonate and ethylene signaling pathway may mediate Fusarium head blight resistance in wheat. Crop Sci. 48:18881896

Maier, F. J., Miedaner, T., Hadeler, B., Felk, A., Salomon, S., Lemmens, M., Kassner, H., and Schäfer, W. 2006. Involvement of trichothecenes in fusarioses of wheat, barley and maize evaluated by gene disruption of the trichodiene synthase (Tri5) gene in three field isolates of different chemotype and virulence. Mol. Plant Pathol. 7:449-461.

Makandar, R., Essig, J. S., Schapaugh, M. A., Trick, H. N., and Shah, J. 2006. Genetically engineered resistance to Fusarium head blight in wheat by expression of Arabidopsis NPR1. Mol. Plant-Microbe Interact. 19:123-129.

Makandar, R., Nalam, V., Chaturvedi, R., Jeannotte, R., Sparks, A. A., and Shah, J. 2010. Involvement of salicylate and jasmonate signaling pathways in Arabidopsis interaction with Fusarium graminearum. Mol. Plant-Microbe Interact. 23:861-870.

Matsuo, H., Taniguchi, K., Hiramoto, T., Yamada, T., Ichinose, Y., Toyoda, K., Takeda, K., and Shiraishi, T. 2001. Gramine increase associated with rapid and transient systemic resistance in barley seedlings induced by mechanical and biological stresses. Plant Cell Physiol. 42:11031111.

Niemeyer, H. M., and Roveri, O. A. 1984. Effects of gramine on energy metabolism of rat and bovine mitochondria. Biochem. Pharmacol. 33:2973-2979.

Overland, L. 1966. The role of allelopathic substances in the "smother crop" barley. Am. J. Bot. 53:423-432.

Parry, D. W., Jenkinson, P., and McLeod, L. 1995. Fusarium ear blight 
(scab) in small-grain cereals-a review. Plant Pathol. 44:207-238.

Pitzschke, A., Schikora, A., and Hirt, H. 2009. MAPK cascade signalling networks in plant defence. Curr. Opin. Plant Biol. 12:421-426.

Pritsch, C., Muehlbauer, G. J., Bushnell, W. R., Somers, D. A., and Vance, C. P. 2000. Fungal development and induction of defense response genes during early infection of wheat spikes by Fusarium graminearum. Mol. Plant-Microbe Interact. 13:159-169.

Proctor, R. H., Hohn, T. M., and McCormick, S. P. 1995. Reduced virulence of Gibberella zeae caused by disruption of a trichothecene toxin biosynthetic gene. Mol. Plant-Microbe Interact. 8:593-601.

Savitch, L. V., Subramaniam, R., Allard, G. C., and Singh, J. 2007. The GLK1 'regulon' encodes disease defense related proteins and confers resistance to Fusarium graminearum in Arabidopsis. Biochem. Biophys. Res. Commun. 359:234-238.

Schreiber, K., and Desveaux, D. 2008. Message in a bottle: Chemical biology of induced disease resistance in plants. Plant Pathol. J. 24:245-268.

Schreiber, K., Ckurshumova, W., Peek, J., and Desveaux, D. 2008. A highthroughput chemical screen for resistance to Pseudomonas syringae in Arabidopsis. Plant J. 54:522-531.

Schwessinger, B., and Zipfel, C. 2008. News from the frontline: Recent in- sights into PAMP-triggered immunity in plants. Curr. Opin. Plant Biol 11:389-395.

Sinha, R. C., Savard, M. E., and Lau, R. 1995. Production of monoclonal antibodies for the specific detection of deoxynivalenol and 15-acetyldeoxynivalenol by ELISA. J. Agric. Food Chem. 43:1740-1744.

Stockwell, B. R. 2000. Chemical genetics: Ligand-based discovery of gene function. Nat. Rev. Genet. 1:116-125.

Urban, M., Daniels, S., Mott, E., and Hammond-Kosack, K. 2002. Arabidopsis is susceptible to the cereal ear blight fungal pathogens Fusarium graminearum and Fusarium culmorum. Plant J. 32:961-973.

Van Ginkel, M., Van der Schaar, W., Yang, Z. P., and Rajaram, S. 1996. Inheritance of resistance to scab in two wheat cultivars from Brazil and China. Plant Dis. 80:863-867.

Wippich, C., and Wink, M. 1985. Biological properties of alkaloids. Influence of quinolizidine alkaloids and gramine on the germination and development of powdery mildew, Erysiphe graminis f. sp. hordei. Experientia 41:1477-1479.

Zúñiga, G. E., Varanda, E. M., and Corcuera, L. J. 1988. Effect of gramine on the feeding behavior of the aphids Schizaphis graminum and Rhopalosiphum padi. Entomol. Exp. Appl. 47:161-165. 